\title{
Extended Field Radiotherapy With or Without Chemotherapy in Patients with Cervical Cancer and Positive Para-Aortic Lymph Nodes: a Single Institution Retrospective Review
}

\author{
Boon Huat Ng ${ }^{1 *}$, AM Rozita ${ }^{1}$, A Adlinda ${ }^{1}$, Wei Ching Lee², WI Wan Zamaniah ${ }^{1}$
}

\begin{abstract}
Background: Positive para-aortic lymph node (PALN) at diagnosis in cervical cancer patients confers an unfavorable prognosis. This study reviewed the outcomes of extended field radiotherapy (EFRT) and concurrent chemotherapy with extended field RT (CCEFRT) in patients with positive PALN at diagnosis. Materials and Methods: Medical records of 407 cervical cancer patients between 1st January 2002 to 31 st December 2012 were reviewed. Some 32 cases with positive PALN were identified to have received definitive extended field radiotherapy with or without chemotherapy. Treatment outcomes, clinicopathological factors affecting survival and radiotherapy related acute and late effects were analyzed. Results: Totals of 13 and 19 patients underwent EFRT and CCEFRT respectively during the period of review. The median follow-up was 70 months. The 5-year overall survival (OS) was $40 \%$ for patients who underwent CCEFRT as compared to $18 \%$ for patients who had EFRT alone, with median survival sof 29 months and 13 months, respectively. The 5-years progression free survival (PFS) for patients who underwent CCEFRT was 32\% and $18 \%$ for those who had EFRT. Median PFS were 18 months and 12 months, respectively. Overall treatment time (OTT) less than 8 weeks reduced risk of death by $81 \%$ (HR=0.19). Acute side effects were documented in $69.7 \%$ and $89.5 \%$ of patients who underwent EFRT and CCEFRT, respectively. Four patients $(\mathbf{1 2 . 5 \%})$ developed radiotherapy late toxicity and there was no treatment-related death observed. Conclusions: CCEFRT is associated with higher 5-years OS and median OS compared to EFRT and with tolerable level of acute and late toxicities in selected patients with cervical cancer and PALN metastasis.
\end{abstract}

Keywords: Cervical carcinoma - concurrent chemo radiation - extended field radiotherapy

Asian Pac J Cancer Prev, 16 (9), 3827-3833

\section{Introduction}

Cervical cancer is the fourth most common cancer affecting women worldwide, with 528,000 new cases diagnose every year. Also, with 266,000 deaths in 2012, it is the fourth most common cause of cancer death in women worldwide (Ferlay et al., 2013). At the Pacific Region, the age standardized incident and mortality rate range from 8.2 to 50.7 and 2.7 to 23.9 per 100,000 womenper year respectively (Obel J et al., 2014). In Peninsular Malaysia (2003-2005), cervical cancer is the second most common cancer among women, constituting $10.6 \%$ of all female cancer with age-standardized incidence of 16.1 per 100,000 women (GCC Lim et al., 2008).

There is a subset of cervical cancer patients who present with para-aortic lymph node (PALN) metastasis during initial diagnosis either detected clinically (radiological features) or surgically (pathologic confirmation). The progressive increase in the prevalence of PALN metastasis was noted with more advanced International Federation of Gynecology and Obstetrics (FIGO) stages; 5\%, 16\% and
$25 \%$ for FIGO stage IB, II and III respectively (Berman et al., 1984). The presence of PALN metastasis confers an unfavorable prognosis. In addition, the presence or absence of PALN metastasis outweighs FIGO staging as prognostic factor (Grigsby et al., 2001). Within those with PALN metastasis, prognostic factors identified to affect overall survival were patient's age, diameter of primary lesion and number of lymph nodes metastasis (Alvarez et al., 1989).

For cervical cancer patients with positive PALN at the time of initial diagnosis, more need to be done to ensure optimal treatment aiming at cure. Several studies as shown in Table 1, reported encouraging results for treatment of PALN metastasis with extended field radiotherapy (EFRT) with 5-years overall survival (OS) ranging from $29 \%-50 \%$ and with tolerable side effects (Nori et al., 1985; Lovecchio et al., 1989; Podczaski et al., 1990; Stryker et al., 2000). These studies encouraged more investigations into multi-modalities treatment to improve the results further. The impact of concurrent chemotherapy and extended field RT (CCEFRT) had been

${ }^{1}$ Clinical Oncology Unit, Faculty of Medicine, University of Malaya, Kuala Lumpur, ${ }^{2}$ Department of Clinical Oncology and Radiotherapy, Penang General Hospital, Penang, Malaysia*For correspondence: ngbh88@yahoo.com 
studied in few trials, with varying success, also shown in Table 1 (Varia et al., 1998; Grigsby et al., 2001; Small et al., 2007; Rajasooriyar et al., 2011). In Asia, few studies started to report encouraging results with CCEFRT in isolated para-aortic lymph node recurrence after definitive irradiation for cervical cancer. Five years OS involving 19, 46 and 33 patients were reported by Chou et al. (2001) Hong et al. (2004) and Kim et al. (2009) respectively, to be in the range of $27 \%-51 \%$. A proportion of patients who underwent CCEFRT managed to achieve long term remission. This is because not all distant metastasis to PALN equals to having systemic disease. Studies also confirmed the safety of this treatment and acceptable late toxicities despite increased acute toxicities (Varia et al., 1998).

However, there is lack of data comparing the treatment outcomes between EFRT and CCEFRT in cervical cancer patients with initial PALN metastasis. With that in mind, we examined the clinicopathological factors associated with survival, treatment related side effects and pattern of relapse in this group of patients treated with either EFRT or CCEFRT.

\section{Materials and Methods}

\section{Patients}

We included in this retrospective review all newly diagnosed FIGO stage 1 to IVA invasive carcinoma of the cervix with PALN metastasis treated with EFRT or CCEFRT at Clinical Oncology Unit, University Malaya Medical Centre (UMMC) between 1st January 2002 to 31st December 2012. A total of 407 medical records of cervical cancer patients undergoing radiotherapy during this period were reviewed with 32 cases identified and included in this analysis. Positive PALN metastasis was confirmed pathologically after surgical resection or based on radiographic criteria $(>1.0 \mathrm{~cm}$ in short axis dimension, multiplicity or heterogeneous enhancement) at the time of diagnosis.

Patients with invasive squamous cell carcinoma, adenosquamous carcinoma or adenocarcinoma of cervix were included while other histology was excluded. Patients were excluded if they had distant metastasis, synchronous malignancies or treated with palliative intent. Data collected included patient's demographics,

Table 1. Studies of Treatment for Cervical Cancer with Para-Aortic Lymph Node Metastases Treated with Extended Field Radiotherapy (EFRT) or Concurrent Chemotherapy with Extended Field Radiotherapy (CCEFRT)

\begin{tabular}{lccc}
\hline Study & $\begin{array}{c}\text { No. of } \\
\text { patients }\end{array}$ & Treatment & $\begin{array}{c}\text { Overall survival } \\
\text { (months) }\end{array}$ \\
\hline D. Nori et al., 1985 & 31 & EFRT & 5-years: 29\% \\
J.L. Lovecchio et al., 1989 & 36 & EFRT & 5-years: 50\% \\
Podczaski E. et al., 1990 & 33 & EFRT & 5-years: 31\% \\
Stryker JA, et al., 2000 & 35 & EFRT & 5-years: 29\% \\
Varia et al., 1998 & 86 & CCEFRT & 3-years: 39\% \\
P.W. Grigsby et al., 2001 & 30 & CCEFRT & 4-years: 29\% \\
W. Small Jr. et al., 2007 & 26 & CCEFRT & 18-months: 60\% \\
Rajasooriyar C et al., 2011 & 39 & CCEFRT & 5-years: 26\% \\
\hline
\end{tabular}

clinicopathological factors with clinical stage based on FIGO staging, treatment details and outcomes, details of cervical cancer relapse and side effects of treatment.

\section{Treatments}

Pretreatment workout for all patients included full history, physical examination, staging computed tomography (CT), pathologic review and blood investigations (complete blood count, renal profile and liver function test). The decision on definitive treatment, either EFRT or CCEFRT was made at the discretion of the treating radiation oncologists taking into account patients performance status and co-morbidities.

\section{Radiotherapy}

Patients were treated with a combination of external beam radiotherapy (EBRT) and intracavitary brachytherapy. The EBRT to the pelvis was either 2D or 3D-planned with conformal four-field box technique and treated using $10 \mathrm{MV}$ photons. In the cases of bulky tumor with posterior and anterior extent of disease towards rectum and/or bladder, an AP/PA two fields technique prescribed to mid-plane dose was used. The superior border was set at L5-S1 interspace while the inferior border was set below the obturator foramen or $3.0 \mathrm{~cm}$ inferior to distal disease, whichever is lower. The lateral borders were located $1.5 \mathrm{~cm}$ outside the bony pelvic sidewalls. For lateral fields, anterior margin is placed at the anterior third of pubic symphysis and posterior border is placed at $\mathrm{S} 2$ and $\mathrm{S} 3$ junction or $3.0 \mathrm{~cm}$ anterior and posterior to distal disease respectively. The superior border of para-aortic strip was set at level T12 to L1 interspace and the inferior border was matched to the superior border of the pelvic portal. The width of the para-aortic portal is usually $8.0 \mathrm{~cm}$ with kidneys excluded. Treatment was with APPA two fields technique prescribed to mid-plane dose or isocentre. The EBRT was given to the pelvis with a standard EBRT dose of $45.0 \mathrm{~Gy}$ to $48.6 \mathrm{~Gy}$ in 25 to 27 fractions in 5 to $5 \frac{1}{2}$ weeks while the PALN region receives a standard EBRT dose of 45Gy in 25 fractions for 5 weeks. Few patients with parametrial involvement had RT boost to pelvis up to $50.4 \mathrm{~Gy}$ to $54.0 \mathrm{~Gy}$ in 28 to 30 fractions in $51 / 2$ to 6 weeks with central shield introduced for the last 3 fractions. Patients treated prior to August 2007 received 20Gy to 40Gy tumor boost to point A using low dose rate (LDR) brachytherapy while those treated after this date received $14.0 \mathrm{~Gy}$ to $22.5 \mathrm{~Gy}$ in 2 to 3 fractions to point $\mathrm{A}$ using high dose rate (HDR) brachytherapy.

\section{Chemotherapy}

Patients who had concurrent chemotherapy received weekly intravenous cisplatin of $40 \mathrm{mg} / \mathrm{m}^{2}$ during radiotherapy treatment.

\section{Follow-up}

After completion of treatment, the follow-up frequency in the beginning was 1-3 months for the initial 6 months, 3-4 months subsequently until 2 years after treatment and thereafter, 6 monthly. Acute and late toxicities were evaluated using Common Terminology Criteria for Adverse Events (CTCAE v4.0) and Radiation Therapy 
Extended Field Radiotherapy and Chemotherapy in Patients with Cervical Cancer and Positive Para-Aortic Lymph Nodes

Oncology Group (RTOG) Common Toxicity Criteria respectively.

\section{Statistical analysis}

Treatment outcomes analyzed were overall survival (OS), cause-specific survival (CSS), median survival (MS), progression free survival (PFS) and loco-regional control (LRC) rate for patients who underwent EFRT and CCEFRT. Clinicopathological factors affecting survival and radiotherapy-related acute and late effects were also analyzed. Patients who were lost to follow-up were contacted via phone to determine their condition. National Registration Department (NRD) were referred to in order to determine survival status for those uncontactable or status unknown patients.

Statistical analysis was performed using SPSS v18.0 software. Survival outcomes were analyzed using the Kaplan-Meier method and compared by log rank test. Univariate and multivariate analysis was used to analyze clinicopathologic factors and treatment details affecting survival.

\section{Results}

\section{Patient clinicopathologic features}

Between 1st January 2002 and 31st December 2012, 32 patients were treated with EFRT and CCEFRT for cervical cancer with PALN metastasis in Clinical Oncology Unit, UMMC. Majority of these patients were in the 50-60 years age group (43.8\%), with a mean age of 55 years (41-86 years). The Chinese was the predominant race presenting with this disease during this period. Majority of the patients $(68.8 \%)$ were of good performance status, (Eastern Cooperative Oncology Group Performance Status) ECOG 0-1 while 31.2\% had ECOG 2. Late presentation was commonly observed, with $75 \%$ presented as locally advanced (FIGO stage II, III and IVA). Squamous cell carcinoma constitutes $87.4 \%$ of cases. All of the patients had been diagnosed with para-aortic nodes metastases either by radiologically or surgically with pathological confirmation.

\section{Treatment details}

EFRT alone was given to 13 patients $(40.6 \%)$ while 19 patients $(59.4 \%)$ underwent CCEFRT. For those who underwent CCEFRT, there were 16 patients $(84.2 \%)$ who completed at least 4 cycles of weekly cisplatin, with minimum and maximum number of cycles of 3 and 6 respectively and mean of 5 cycles weekly cisplatin. The stipulated overall treatment time (OTT) was 8 weeks and $78.1 \%$ managed to complete their RT within this time period. Neo-adjuvant chemotherapy prior to RT was administered to 4 patients $(12.5 \%)$ while 3 patients $(9.4 \%)$ had adjuvant chemotherapy after RT.

\section{Survival analysis}

The median follow-up was 70 months. There were 26 patients analyzed for 5-year survival as their treatment completed by 31 st December 2009. The 5-year overall survival (OS) were $40 \%$ for patients who underwent CCEFRT as compared to $18 \%$ for patients who had
EFRT alone, with median survival of 29 months $(95 \%$ CI, 0 -60.6) and 13 months (95\% CI 0-43.2) for both CCEFRT and EFRT respectively, as shown in Figure 1. The 5-years progression free survival (PFS) for patients who underwent CCEFRT was $32 \%$ and $18 \%$ for those who had EFRT. Median PFS were 18 months $(95 \%$ CI, $13-23)$ and 12 months (95\% CI, 9.3-26.7) respectively, as shown in Figure 2.

The multivariate analysis for survival according to FIGO stage, type of treatment received, performance status (ECOG) and overall treatment time (OTT) are presented in Table 2. In view of the small sample size, survival analysis for FIGO stage is grouped into FIGO I, II and FIGO III, IVA while performance status is grouped into ECOG 0, 1 and ECOG 2. Only overall treatment time (OTT) was found to correlate with survival while type of treatment received, FIGO stage and performance status (ECOG) does not. OTT less than 8 weeks reduces risk of death by $81 \%(\mathrm{HR}=0.19)$.

The 5-years CSS for FIGO stage I and II group of patients was $54 \%$, and for patients with FIGO III and IVA

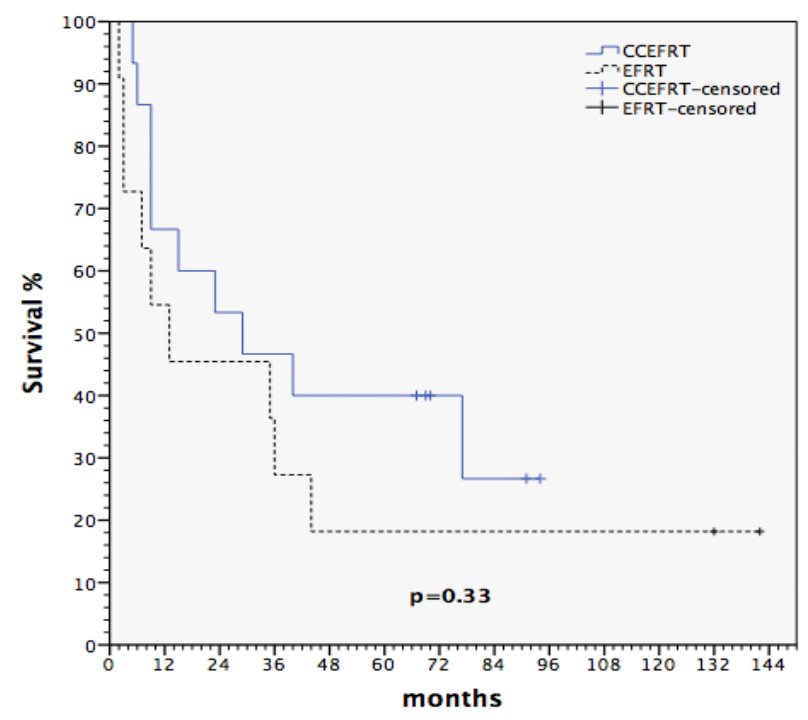

Figure 1. Overall Survival (OS) According to Treatment Received

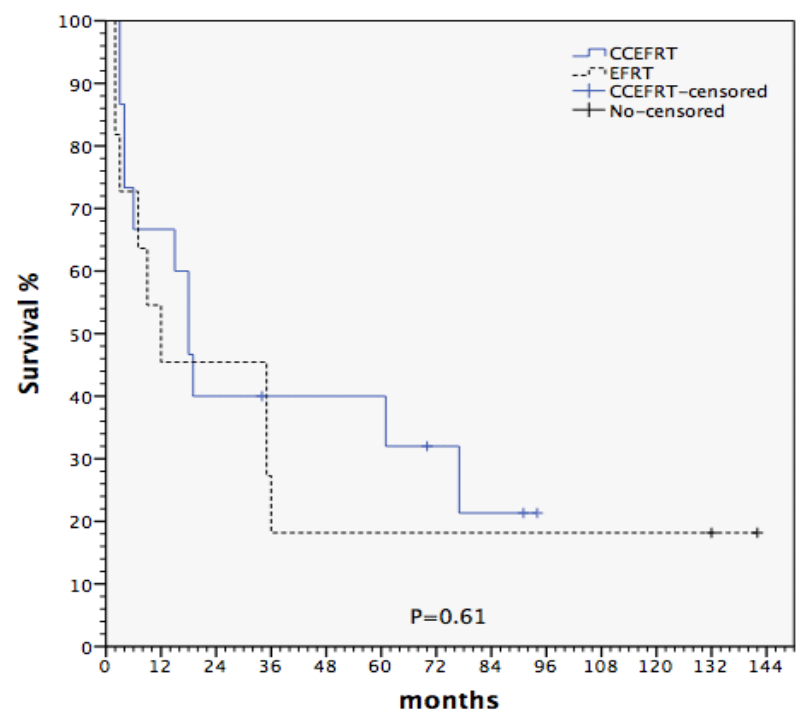

Figure 2. Progression Free Survival (PFS) According to Treatment Received 
Table 2. Multivariate Analysis on Risk of Death Based on FIGO Stage, Treatment Received, ECOG PS and OTT

\begin{tabular}{lccc}
\hline Variables & p-value & HR & 95\% CI \\
\hline FIGO stage (I, II vs III,IV) & 0.31 & 0.82 & $0.30-1.46$ \\
Type of treatment received & 0.56 & 0.79 & $0.35-1.77$ \\
(CCEFRT vs EFRT) & & & \\
ECOG PS (0, 1 vs 2) & 0.12 & 0.53 & $0.22-1.27$ \\
OTT $(<8$ weeks vs $\geq 8$ weeks) & 0.01 & 0.19 & $0.02-0.38$ \\
\hline
\end{tabular}

*EFRT: extended field radiotherapy, CCEFRT: concurrent chemotherapy and extended field radiotherapy, ECOG: Eastern Cooperative Oncology Group Performance Status, FIGO: International Federation of Gynecology and Obstetrics clinical staging for cervical cancer, OTT: overall treatment time

Table 3. Treatment Response and Patterns of Recurrence

\begin{tabular}{lcc}
\hline Variables & No of patients & $\%$ \\
\hline Post treatment tumor response & & \\
Clinical Complete Response (cCR) & 19 & 59.3 \\
Clinical Partial Response (cPR) & 6 & 18.8 \\
Refractory & 7 & 21.9 \\
Patterns of recurrence & 12 & 37.5 \\
No recurrence* & 13 & 40.6 \\
Recurrence & 5 & 15.6 \\
$\quad$ Locoregional & 8 & 25 \\
\hline Distance & 8 \\
\hline
\end{tabular}

*7 patients with pathologically confirmed PALN metastasis and 5 patients based on radiological features of PALN metastasis

was $26 \%$. Overall, 5 -years cause specific survival (CSS) was $35 \%$ and the 5-years loco-regional control (LRC) rate was $74 \%$.

\section{Pattern of failure}

When patterns of failure were reviewed, seven patients $(21.9 \%)$ were found to have residual tumor (1 patient was from FIGO stage I, 5 patients were of FIGO stage III and 1 was patient from FIGO IVA). From the remaining 25 patients, 19 patients (59.3\%) had clinical complete response (cCR) while six patients $(18.8 \%)$ achieved clinical partial response (cPR) by clinical examinations during regular follow-up. At the end of study period, 12 (37.5\%) patients were still in remission while the remaining 13 patients $(40.6 \%)$ had recurrence of disease. These results are shown in Table 3. The commonest pattern of recurrence was distant recurrence affecting $61.5 \%(8 / 13)$ of patients; 3 lung metastasis, 3 supraclavicular lymph node metastasis and 1 each for bone and peritoneum metastasis. The median survival for those who developed distance recurrence was 5.1 months (2.3 - 10.9 months). As for loco-regional recurrence, three patients had relapsed at para-aortic region while two patients recurred locally in the pelvis. Among the five patients who developed loco-regional recurrence, three patients underwent chemoradiotherapy for the para-aortic field (all FIGO stage III) while two patients (both FIGO stage $1 \mathrm{~B}$ ) underwent para-aortic radiation alone. The median survival for those who developed loco-regional recurrence was 7.2 months (5.0-12.8 months). For FIGO stage I, two patients developed local recurrence and one patient developed distant metastasis. As for FIGO stage II, no patient had local recurrence while two patients developed distant recurrence. Three patients each developed local and distant recurrence respectively for FIGO stage III. One patient who was diagnosed FIGO stage IVA had refractory disease despite treatment.

\section{Adverse events}

Acute toxicities were observed in $69.7 \%$ of patients who underwent EFRT treatment and $89.5 \%$ who underwent CCEFRT treatment. Four patients (12.5\%) developed radiotherapy late toxicities with two patients had recto-vaginal fistula, one patient developed small bowel obstruction needing surgical resection and one patient experienced radiation cystitis. All late effects from radiotherapy happened in two patients from each group of treatment, 2/13 (13.4\%) in EFRT and 2/19 (10.5\%) in CCEFRT group. There was no treatment-related death (TRD) observed.

\section{Discussion}

In 1999, five randomized clinical trials incorporating platinum-based chemotherapy to radiation therapy showed an overall survival benefit in patients with stages IB2 to IVA disease (Keys et al., 1999; Morris et al., 1999; Rose et al., 1999; Whitney et al., 1999; Peters et al., 2000). However, for the subset of patients with FIGO stages I to IVA with PALN metastasis, the treatment and outcome reports have been very scarce. We looked into this particular subset of patients and found that successful treatment of the primary disease and PALN metastasis can result in long term remission. This study showed 37.5\% (12 patients; 7 patients with pathologically confirmed PALN metastasis and 5 patients based on radiological features of PALN metastasis) achieved long-term remission. Results of this series also showed an improvement of 5-years OS for CCEFRT from $18 \%$ to $40 \%, 5$-years PFS from $18 \%$ to $32 \%$ and median OS from 13 months to 29 months when compared to EFRT. These results obtained from local data were comparable with what was reported by other studies worldwide, which reported 5-years OS ranging from 26\% to $60 \%$ (Varia et al., 1998; Grigsby et al., 2001; Chou HH et al., 2001; Hong et al., 2004; Small et al., 2007; Kim YS et al., 2009; Rajasooriyar et al., 2011). Notably, local data for cervical cancer patients from FIGO stage 1B2 IVA but without positive paraaortic lymph node reported 5 -year OS and 5-year PFS of 50\% and 48\%, respectively (Wan Zamaniah et al., 2014).

In this study, overall treatment time less than 8 weeks had been shown to correlate positively with overall survival, mirroring study by Petereit DG et al., which pointed out further that survival decreased by $0.6 \% /$ day and pelvic control decreased by $0.7 \% /$ day for each additional day of treatment beyond 55 days (Petereit et al., 1995). KrusunS et al.,also noted significant increase in survival for those completing treatment without interruption while highlighting the main cause of treatment interruption, which were hematologic toxicities for those who underwentconcurrent chemoradiation (Krusun et al., 2014). Type of treatment received, FIGO stage and 
performance status were not found to be significant.

The safety and effectiveness of concurrent chemotherapy with weekly cisplatin $40 \mathrm{mg} / \mathrm{m}^{2}$ had been proven in a metaanalysis by Yan Hu et al. (2012) while concurrent chemotherapy with weekly cisplatin $40 \mathrm{mg} /$ $\mathrm{m}^{2}$ and EFRT of $45 \mathrm{~Gy}$ in $1.8 \mathrm{~Gy}$ fractions had also been proven safe and effective in a study by Rajasooriyar et al. (2011). There were 59.4\% (19 of 32 patients) who successfully achieved complete response to initial treatment, out of which $63.2 \%$ (12 of 19 patients) and $53.8 \%$ (7 of 13 patients) were in CCEFRT and EFRT group respectively. The para-aortic region was prescribed with $45 \mathrm{~Gy}$ in this study. Optimal treatment of PALN metastasis depends on the RT dose prescribed and taking into account prognostic factors such as size, number of PALN metastasis and location of PALN metastasis (Jang et al., 2013). In addition, study by Szu-Yuan WU et al., 2014 showed that RT dose $>50.4$ Gy to PALN is associated with better disease control. Another study by Rash et al. (2013) noted that decrease in standardized uptake value (SUV) was significantly greater when lymph nodes were treated with RT dose $>54 \mathrm{~Gy}$. Nevertheless, majority of relapse occurred at distance sites, necessitating further investigation into adjuvant systemic treatment (Jensen et al., 2012).

However, giving a higher dose of RT is complicated by the surrounding organs at risk. In this study, acute toxicities were mostly grade 1 or 2 and were manageable, tolerable and transient. Grade 3 or 4 acute and late toxicities were experienced by $9.4 \%$ and $12.5 \%$ of patients respectively. When comparison was made, late toxicity affected $13.4 \%$ and $10.5 \%$ of EFRT and CCEFRT patients respectively in this study. This is probably an underestimation as data were collected retrospectively. Despite higher acute toxicities being reported by Kim YS et al. (2009), CCEFRT achieve higher survival rate with low rate of late toxicities. Furthermore, Kazumoto et al. (2011) was able to deliver a dose greater than 54Gy to positive PALN metastasis using CCEFRT safely to all the patients. Conversely, in a study by Small et al. (2007), CCEFRT was associated with a significant grade 3 or 4 acute $(81 \%)$ and late $(40 \%)$ toxicities. Notably, earlier attempt to increase the dose delivered to positive PALNs by using hyperfractionation resulted in unacceptable high rate of grade 4 late toxicity (17\%) (Grigsby et al., 2001).

With the advent of intensity modulated radiotherapy (IMRT), dose intensification is made possible and safe (Mutic et al., 2003). It was demonstrated that with similar target coverage, normal tissue sparing is superior with IMRT as compared to three-dimensional planning system in the treatment of cervical cancer in dosimetric study (Portelance et al., 2001). Treatment plans were able to deliver 60.0Gy to the PET-positive PALN and 50.0Gy to the PALN and pelvic lymph node beds with acceptable acute and late toxicity (Esthappan et al., 2008). Moving forward, concurrent cisplatin chemotherapy with postoperative EF-IMRT was also proven to be safe with acceptable level of acute and late toxicities (Sood et al., 2002, Zhang et al., 2012). As with CCEFRT using threedimensional planning system, concurrent chemotherapy with EF-IMRT also result in predominant mode of distant failure despite having a good locoregional control (Beriwal et al., 2007).

In this study, $37.5 \%$ of patients were found to have positive PALN post-operatively while $62.5 \%$ of patients were clinically staged with imaging studies. The ability to detect PALN metastasis is an important factor and the emerging roles of PET-CT in recent years are evidenced by its ability to detect abnormal LN regions more than CT scan. (Grigsby et al., 2001). Perez-Medina et al. (2013) reported that FDG-PET has high specificity (94.1\%) and low sensitivity (77.7\%), for the detection of PALN metastasis. Gouy et al. (2013) pointed out that presence and absence of pelvic uptake on PET/CT reflected the rate of $24 \%$ and $9 \%$ pathological positive PA nodes respectively. Unfortunately, in early stage cervical cancer (FIGO IB/II), PET/CT imaging without histological examination of the para-aortic area would overlook $8 \%$ of patients with histological PALN metastasis (Boughanim et al., 2008). Thus, the decision for PET/CT ought to be individualized after taking into account presence or absence of pelvic LN involvement and clinical staging to maximize its detection of PALN metastasis.

The low survival outcome of patients with positive PALN disease prompted a few studies to look into treating para-aortic region prophylactically. However, the rate of PALN micrometastasis in patients with locally advanced cervical cancer is low even with the use of ultrastaging and immunohistochemical analysis (Zand et al., 2010). Furthermore, only patients with high probability of local control seemed to benefit from concomitant weekly cisplatin and prophylactic para-aortic radiation (Haie et al., 1988). The importance of local control can be seen in this study as of the seven patients who remained refractory to treatment, six patients were of FIGO stage III and one patient FIGO stage IVA. Thus, routine prophylactic paraaortic irradiation for all high risk cervical cancer patients without positive para-aortic lymph node is of limited value if unable to achieve local control. Patient selection and individualization of treatment is the key to successful treatment. In another subset of cervical cancer patients who presented with isolated PALN recurrence, current studies suggested that salvage CCEFRT contributed positively towards improvement of overall survival (Singh et al., 2005). In order for early detection of any isolated PALN recurrence, periodical surveillance with clinical examination and imaging studies are of help for early detection and thus initiate the salvage treatment for these group of patients.

In conclusion, treatment with CCEFRT is associated with higher 5-years OS, median OS compared to EFRT and with tolerable level of acute and late toxicities in selected patients with cervical cancer and PALN metastasis. Further studies looking at concurrent chemotherapy with EF-IMRT and incorporating use of adjuvant chemotherapy with hope to reduce distant metastasis are needed to improve the prognosis of cervical cancer patients with PALN metastasis.

\section{References}

Alvarez RD, Soong SJ, Kinney WK, et al (1989). Identification 
of prognostic factors and risk groups in patients found to have nodal metastasis at the time of radical hysterectomy for early-stage squamous carcinoma of the cervix. Gynecol Oncol, 35, 130-5.

Beriwal S, Gan GN, Heron DE, et al (2007). Early clinical outcome with concurrent chemotherapy and extended-field, intensity-modulated radiotherapy for cervical cancer. Int $J$ Radiat Oncol Biol Phys, 68, 166-71.

Berman ML, Keys H, Creasman W, et al (1984). Survival and patterns of recurrence in cervical cancer metastatic to periaortic lymph nodes (a gynecologic oncology group study). Gynecol Oncol, 19, 8-16.

Boughanim M, Leboulleux S, Rey A, et al (2008). Histologic results of para-aortic lymphadenectomy in patients treated for stage IB2/II cervical cancer with negative [18F] fluorodeoxyglucose positron emission tomography scans in the para-aortic area. J Clin Oncol, 26, 2558-61.

Chou HH, Wang CC, Lai CH, et al (2001). Isolated paraaortic lymph node recurrence after definitive irradiation for cervical carcinoma. Int J Radial Oncol Biol Phys, 51, 442-8.

Esthappan J, Chaudhari S, Santanam L, et al (2008). Prospective clinical trial of positron emission tomography/computed tomography image-guided intensity-modulated radiation therapy for cervical carcinoma with positive para-aortic lymph nodes. Int J Radiat Oncol Biol Phys, 72, 1134-9.

Ferlay J, Soerjomataram I, Ervik M, et al (2013). GLOBOCAN 2012 v1 .0, cancer incidence and mortality worldwide: IARC CancerBase No. 11 [Internet]. Lyon, France: international agency for research on cancer; 2013.

GCC Lim, Y Halimah, S Rampal (2007). Cancer incidence in Peninsular Malaysia, 2003-2005. the third report of the national cancer registry, Malaysia.

Gouy S, Morice P, Narducci F, et al (2013). Prospective multicenter study evaluating the survival of patients with locally advanced cervical cancer undergoing laparoscopic para-aortic lymphadenectomy before chemoradiotherapy in the era of positron emission tomography imaging. J Clin Oncol, 31, 3026-33.

Grigsby PW, Heydon K, Mutch DG, et al (2001a). Long-term follow-up of RTOG 92-10: Cervical cancer with positive para-aortic lymph nodes. Int J Radiat Oncol Biol Phys, 51, 982-7.

Grigsby PW, Perez CA, Chao KS, et al (2001b). Radiation therapy for carcinoma of the cervix with biopsy-proven positive para-aortic lymph nodes. Int J Radiat Oncol Biol Phys, 49, 733-8.

Haie C, Pejovic MH, Gerbaulet A, et al (1988). Is prophylactic para-aortic irradiation worthwhile in the treatment of advanced cervical carcinoma? Results of a controlled clinical trial of the EORTC radiotherapy group. Radiother Oncol, 11, 101-12.

Hong JH, Tsai CS, Lai CH, et al (2004). Recurrent squamous cell carcinoma of cervix following definitive radiotherapy. Int J Radiat Oncol Biol Phys, 60, 249-57.

Hu Y, Cai ZQ, Su XY (2012). Concurrent weekly cisplatin versus triweekly cisplatin with radiotherapy in the treatment of cervical cancer: a meta-analysis result. Asian Pac J Cancer Prev, 13, 4301-4.

Jang H, Chun M, Cho O, et al (2013). Prognostic factors and treatment outcome after radiotherapy in cervical cancer patients with isolated para-aortic lymph node metastases. $J$ Gynecol Oncol, 24, 229-35.

Jensen LG, Hasselle MD, Rose BS, et al (2013). Outcomes for patients with cervical cancer treated with extendedfield intensity-modulated radiation therapy and concurrent cisplatin. Int J Gynecol Cancer, 23, 119-25.

Kazumoto T, Kato S, Yokota H, et al (2011). Is a low dose of concomitant chemotherapy with extended-field radiotherapy acceptable as an efficient treatment for cervical cancer patients with metastases to the para-aortic lymph nodes? Int J Gynecol Cancer, 21, 1465-71

Keys HM, Bundy BN, Stehman FB, et al (1999). Cisplatin, radiation, and adjuvant hysterectomy compared with radiation and adjuvant hysterectomy for bulky stage IB cervical carcinoma. N Engl J Med, 340, 1154-61.

Kim YS, Kim JH, Ahn SD, et al (2009). High-dose extended-field irradiation and high-dose-rate brachytherapy with concurrent chemotherapy for cervical cancer with positive para-aortic lymph nodes. Int J Radiat Oncol Biol Phys, 74, 1522-8.

Krusun S, Pesee M, Supakalin N, et al (2014). Treatment interruption during concurrent chemoradiotherapy of uterine cervical cancer; analysis of factors and outcomes. Asian Pac J Cancer Prev, 15, 5653-7.

Lovecchio JL, Averette HE, Doinato D, et al (1989). 5-Year survival of patients with periaortic nodal metastases in clinical stage IB and II cervical carcinoma. Gynecol Oncol, 34, 43-5.

Morris M, Eifel PJ, Lu J, et al (1999). Pelvic radiation with concurrent chemotherapy compared with pelvic and paraaortic radiation for high risk cervical cancer. $N$ Eng J Med, 340, 1137-43.

Mutic S, Malyapa RS, Grigsby PW, et al (2003). PET-guided IMRT for cervical carcinoma with positive para-aortic lymph nodes-a dose-escalation treatment planning study. Int J Radiat Oncol Biol Phys, 55, 28-35.

Nori D, Valentine E, Hilaris B (1985). The role of paraaortic node irradiation in the treatment of cancer of the cervix. Int $J$ Radiat Oncol Biol Phys, 211, 1469-73.

Obel J, Souares Y, Hoy D, et al (2014). A systematic review of cervical cancer incidence and mortality in the pacific region. Asian Pac J Cancer Prev, 15, 9433-7.

Perez-Medina T, Pereira A, Mucientes J, et al (2013). Prospective evaluation of 18-fluoro-2-deoxy-D-glucose positron emission tomography for the discrimination of paraaortic nodal spread in patients with locally advanced cervical carcinoma. Int J Gynecol Cancer, 23, 170-5.

Petereit DG, Sarkaria JN, Chappell R, et al (1995). The adverse effect of treatment prolongation in cervical carcinoma. Int $J$ Radiat Oncol Biol Phys, 32, 1301-7.

Peters WA, Liu PY, Barrett RJ, et al (2000). Concurrent chemotherapy and pelvic radiation therapy compared with pelvic radiation therapy alone as adjuvant therapy after radical surgery in high-risk early-stage cancer of the cervix. J ClinOncol, 18, 1606-13.

Podczaski E, Stryker JA, Kaminski P, et al (1990). Extendedfield radiation therapy for carcinoma of the cervix. Cancer, 66, 251-8.

Portelance L, Chao KS, Grigsby PW, Bennet H, Low D (2001). Intensity-modulated radiation therapy (IMRT) reduces small bowel, rectum, and bladder doses in patients with cervical cancer receiving pelvic and para-aortic irradiation. Int $J$ Radiat Oncol Biol Phys, 51, 261-6.

Rajasooriyar C, Van Dyk S, Bernshaw D, et al (2011). Patterns of failure and treatment-related toxicity in advanced cervical cancer patients treated using extended field radiotherapy with curative intent. Int J Radiat Oncol Biol Phys, 80,422-8.

Rash DL, Lee YC, Kashefi A, et al (2013). Clinical response of pelvic and para-aortic lymphadenopathy to a radiation boost in the definitive management of locally advanced cervical cancer. Int J Radiat Oncol Biol Phys, 87, 317-22.

Rose PG, Bundy BN, Watkins EB, et al (1999). Concurrent cisplatin-based radiotherapy and chemotherapy for locally advanced cervical cancer. $N$ Eng J Med, 340, 1144-53.

Small W, Winter K, Levenback C, et al (2007). Extended-field 
irradiation and intracavitary brachytherapy combined with cisplatin chemotherapy for cervical cancer with positive para-aortic or high common iliac lymph nodes: Results of arm 1 of RTOG 0116. Int J Radiat Oncol Biol Phys, 68, 1081-7.

Sood BM, Timmins PF, Gorla GR, et al (2002). Concomitant cisplatin and extended field radiation therapy in patients with cervical and endometrial cancer. Int J Gynecol Cancer, 12, 459-64.

Stryker JA, Mortel R (2000). Survival following extended field irradiation in carcinoma of cervix metastasis to para-aortic lymph nodes. Gynecol Oncol, 79, 399-405.

Szu-Yuan WU, Eng-Yen HUANG, et al (2014). Prognostic factors associated with radiotherapy for cervical cancer with computed tomography-detected para-aortic lymph node metastasis. J Radiat Res, 55, 129-38.

Varia MA, Bundy BN, Deppe G, et al (1998). Cervical carcinoma metastatic to para-aortic nodes: extended field radiation therapy with concomitant 5-fluorouracil and cisplatin chemotherapy: a Gynecologic Oncology Group study. Int $J$ Radiat Oncol Biol Phys, 42, 1015-23.

Whitney CW, Sause W, Bundy BN, et al (1999). Randomized comparison of fluorouracil plus cisplatin vs hydroxyurea as an adjunct to radiation therapy in stages IIB-IVA carcinoma of the cervix with negative para-aortic lymph nodes: Gynecologic Oncology Group and Southwest Oncology Group Study. J Clin Oncol, 17, 1339-48.

Zamaniah WI, Mastura MY, Phua CE, et al (2014). Definitive concurrent chemoradiotherapy in cervical cancer-a University of Malaya Medical Centre experience. Asian Pac J Cancer Prev, 15, 8987-92.

Zand B, Euscher ED, Soliman PT, et al (2010). Rate of paraaortic lymph node micrometastasis in patients with locally advanced cervical cancer. Gynecol Oncol, 119, 422-5.

Zhang G, Fu C, Zhang Y, et al (2012). Extended-field intensitymodulated radiotherapy and concurrent cisplatin-based chemotherapy for postoperative cervical cancer with common iliac or para-aortic lymph node metastases: a retrospective review in a single institution. Int J Gynecol Cancer, 22, 1220-5. 